\title{
Heteronuclear isotropic mixing separated local field NMR spectroscopy
}

\author{
Sergey V. Dvinskikh ${ }^{\text {a),b) }}$ \\ Biophysics Research Division, University of Michigan, Ann Arbor, Michigan 48109-1055, \\ Department of Chemistry, University of Michigan, Ann Arbor, Michigan 48109-1055, \\ and Institute of Physics, St. Petersburg State University, 198504 St. Petersburg, Russia
}

Kazutoshi Yamamoto and Ayyalusamy Ramamoorthy ${ }^{\mathrm{a}), \mathrm{c})}$

Biophysics Research Division, University of Michigan, Ann Arbor, Michigan 48109-1055 and Department of Chemistry, University of Michigan, Ann Arbor, Michigan 48109-1055

(Received 11 April 2006; accepted 17 May 2006; published online 20 July 2006)

\begin{abstract}
This paper presents a theoretical, numerical, and experimental study of a new class of separated local field (SLF) techniques. These techniques are based on the heteronuclear isotropic mixing leading to spin exchange via the local field (HIMSELF). It is shown that highly efficient and robust SLF experiments can be designed based on double channel windowless homonuclear decoupling sequences. Compared to rotating frame techniques based on Hartmann-Hahn cross polarization, the new approach is less susceptible to the frequency offset and chemical shift interaction and can be applied in the structural studies of macromolecules that are uniformly labeled with isotopes such as ${ }^{13} \mathrm{C}$ and ${ }^{15} \mathrm{~N}$. Furthermore, isotropic mixing sequences allow for transfer of any magnetization component of one nucleus to the corresponding component of its dipolar coupled partner. The performance of HIMSELF is studied by analysis of the average Hamiltonian and numerical simulation and is experimentally demonstrated on a single crystalline sample of a dipeptide and a liquid crystalline sample exhibiting motionally averaged dipolar couplings. () 2006 American Institute of Physics. [DOI: 10.1063/1.2212939]
\end{abstract}

\section{INTRODUCTION}

Due to its dependence on molecular orientation and interatomic distance, the through-space dipole-dipole coupling represents a very important spin interaction in nuclear magnetic resonance (NMR) spectroscopy. The dipolar coupling is also sensitive to dynamics, which means that this interaction can report on molecular conformation and motion at the atomic level. In this work, we focus on measuring the heteronuclear dipolar couplings between abundant (typically ${ }^{1} \mathrm{H}$ ) and rare (typically ${ }^{13} \mathrm{C}$ or ${ }^{15} \mathrm{~N}$ ) nuclear spins in macroscopically aligned materials such as single crystal solids or oriented liquid crystalline and aligned biological samples. There exist today a wide range of solid-state NMR methods, generally referenced as separated local field (SLF) spectroscopy, capable of estimating these informative couplings. ${ }^{1-5}$ One of the most powerful approaches, popular in structural biological studies, is based on the exchange of spin polarizations in the rotating frame. ${ }^{6}$ The advantages of using rotating frame techniques include (i) a large heteronuclear dipolar scaling factor which facilitates the measurement of small dipole-dipole interactions, (ii) simple spectral shape, and (iii) high dipolar resolution.

We recently proposed ${ }^{7,8}$ a number of new rotating frame SLF techniques based on isotropic mixing of spin components using the previously developed multiple pulse homonuclear decoupling sequences. ${ }^{9,10}$ Similar to spin exchange

\footnotetext{
a) Authors to whom correspondence should be addressed.

${ }^{b)}$ Electronic mail: sdvinskikh@mail.ru

${ }^{c)}$ Electronic mail: ramamoor@umich.edu
}

experiments, ${ }^{6}$ the new approach employs polarization exchange in the rotating frame, but allows for isotropic mixing, meaning that an arbitrary magnetization component of one spin can be transferred to the corresponding component of the dipolar coupled partner. This adds a new degree of flexibility to the design of multidimensional experiments, where a coherent polarization transfer is needed. Moreover, new sequences are less sensitive to the interfering contributions from the chemical shift and frequency resonance offset and can be applied to the isotopically uniformly labeled samples.

In this work, we present a theoretical analysis, numerical simulation, and experimental study of techniques that are based on the heteronuclear isotropic mixing leading to spin exchange via the local field (HIMSELF). We analyze the performance of the method in terms of the susceptibility to rf field imperfections and the sensitivity to resonance frequency offset and chemical shift interactions and compare with other techniques.

\section{PULSE SEQUENCES}

A schematic pulse sequence for two-dimensional (2D) SLF spectroscopy using the double channel homonuclear decoupling sequences of Burum, Linder, Ernst, and Waugh $(\mathrm{BLEW})^{9}$ or windowless isotropic mixing (WIM) ${ }^{10}$ type during the variable evolution period $t_{1}$ is shown in Fig. 1(a). Initial magnetizations of abundant nuclei (with spin $I$ ) are prepared by cross polarization ${ }^{11}(\mathrm{CP})$ and a tilt pulse with a flip angle of $\vartheta$. The heteronuclear dipolar coupling is monitored through the oscillation of the $S$ (rare) spin polarization in the rotating frame resulting from the coherent polarization 
a)

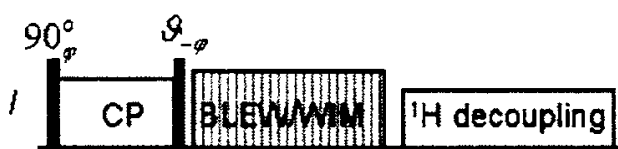

$S$

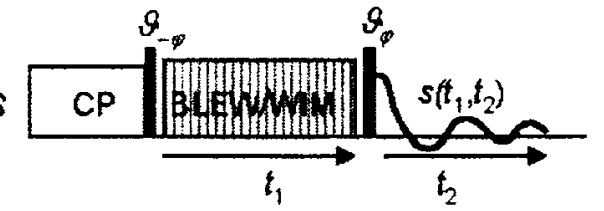

b)

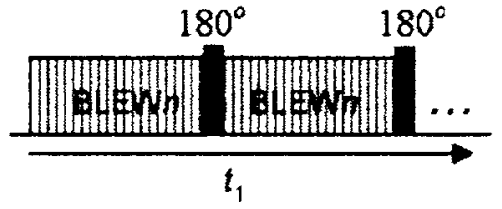

FIG. 1. (a) A two-dimensional HIMSELF sequence using BLEWn ( $n$ $=12,48$ ) or WIM24 to measure the heteronuclear dipolar couplings in oriented static samples. The initial magnetizations of nuclei $I$ are prepared using cross polarization and a tilt pulse with a flip angle of $\vartheta$. Homonuclear decoupling sequences BLEW12, BLEW48, or WIM24 are applied in both the radio frequency channels synchronously in the evolution period during the incremented time $t_{1}$. The signal is detected during $t_{2}$ by decoupling nuclei $I$. (b) Broadband sequences BB-BLEW $n$ are obtained by inserting $180^{\circ}$ pulses between BLEW $n$ rf cycles. The phase of $180^{\circ}$ pulses is, in the rotating frame, orthogonal to the effective field direction of BLEWn. These broadband sequences suppress offset effects as discussed in the text.

exchange between $I$ and $S$ spins during $t_{1}$. By incrementing the delay $t_{1}$, the exchange process is encoded in the signal $s\left(t_{1}, t_{2}\right)$ of the $S$ spin recorded during $t_{2}$ period. The homonuclear dipolar spin interactions of both $I$ and $S$ spins are suppressed. Therefore, the method can also be applied to study samples uniformly labeled with isotopes such as ${ }^{13} \mathrm{C}$ or ${ }^{15} \mathrm{~N}$.

While the chemical shift spin interaction is suppressed during WIM irradiation, it is preserved when the BLEW-type sequences are employed. It is possible to suppress the chemical shift effects by inserting the $180^{\circ}$ pulse after each BLEW cycle synchronously in both radio frequency (rf) channels as shown in Fig. 1(b).

\section{THEORETICAL BACKGROUND}

We restrict the theoretical treatment to an isolated twospin system. Abundant (proton) and rare (e.g., ${ }^{13} \mathrm{C}$ or $\left.{ }^{15} \mathrm{~N}\right)$ spins are denoted as $I$ and $S$, respectively. Numerical simulations are used to treat spin clusters containing more than one $I$ spin (see Sec. V).

The high-field truncated spin Hamiltonian during the evolution period, $t_{1}$, in the doubly rotating frame, can be written as

$$
H=H_{\mathrm{rf}}+H_{\mathrm{int}},
$$

where $H_{\mathrm{rf}}(t)$ represents the rf irradiation, and the spin interaction Hamiltonian is given by

$$
H_{\mathrm{int}}=\omega_{I S} 2 I_{z} S_{z}+\Omega_{I} I_{z}+\Omega_{S} S_{z} .
$$

Here, the first term represents the heteronuclear $I-S$ interaction $H_{I S}$ with a coupling constant of $\omega_{I S}$, and the second and third terms are the frequency offset/chemical shift terms in $H_{I}$ and $H_{S}$. The heteronuclear coupling constant $\omega_{I S}$ consists

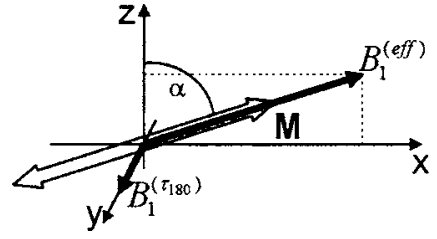

FIG. 2. The direction of the effective rf field of the BLEW12 sequence. Spin magnetization $M$ is prepared along the $B_{1}^{\text {(eff) }}$ field direction. By applying the $180_{y}^{\circ} \mathrm{rf}$ pulse, the magnetization direction is inverted.

of the heteronuclear dipolar coupling $2 \pi d_{I S}$ and the indirect $J$ coupling (typically, much smaller), $\pi J_{I S}, \omega_{I S}=2 \pi d_{I S}+\pi J_{I S}$. The Hamiltonian in the toggling frame is obtained by the transformation $^{12}$

$$
\tilde{H}_{\text {int }}(t)=U_{\text {rf }}^{-1}(t) H_{\text {int }} U_{\text {rf }}(t)
$$

using the rf propagator $U_{\mathrm{rf}}(t)=e^{-i H_{\mathrm{rr}} t}$.

In the following, rf irradiation consisting of back-to-back rf pulses (i.e., without windows between pulses) will be considered. For example, BLEWn $(n=12,48)$ sequences are composed of the $90^{\circ}$ pulses. ${ }^{9}$ Hence, the rf propagator $U_{\mathrm{rf}}^{(k)}(t)$, active during the $k$ th pulse, i.e., during the time interval $t \in\left[(k-1) \tau_{90}, k \tau_{90}\right]$, where $\tau_{90}$ is the $90^{\circ}$ pulse width, can be presented as

$$
U_{\mathrm{rf}}^{(k)}(t)=D \exp \left\{i \omega\left[t-(k-1) \tau_{90}\right] I_{\alpha_{k}}\right\} P_{k-1} \cdots P_{2} P_{1},
$$

where the propagator $P_{i}$ is given as

$$
P_{i}=\exp \left[ \pm i(\pi / 2) I_{\alpha_{i}}\right] \text {, }
$$

and it represents a $90^{\circ}$ pulse along $\alpha_{i}=x, y$ axes and $D$ is the Dyson time ordering operator. The zeroth-order average Hamiltonian during the $k$ th pulse is calculated as

$$
\overline{\tilde{H}}_{\mathrm{int}}^{(k)}=\frac{1}{\tau_{90}} \int_{0}^{\tau_{90}}\left(U_{\mathrm{rf}}^{(k)}\right)^{-1} H_{\mathrm{int}} U_{\mathrm{rf}}^{(k)} d t
$$

The zeroth-order average Hamiltonian for a sequence of $n$ pulses is provided by a sum as given below:

$$
\tilde{\tilde{H}}_{\text {int }}\left(\tau_{c}\right)=\frac{1}{\tau_{c k=1}} \sum^{n} \tau_{90} \overline{\tilde{H}}_{\text {int }}^{(k)},
$$

where $\tau_{c}=n \tau_{90}$.

\section{A. BLEW $n$ spin-lock sequences 1. BLEW12 spin lock}

The BLEW12 spin-lock sequence is the BLEW12 irradiation ${ }^{9}$ applied simultaneously and synchronously in $I$ and $S$ rf channels as shown in Fig. 1(a). By using the relationships (3)-(6), the dipolar terms $\tilde{\tilde{H}}_{I S}^{(n)}$ of the spin interaction Hamiltonian $\overline{\tilde{H}}_{\text {int }}^{(n)}$ for the first six rf pulses of BLEW12 spin-lock sequence are calculated to 


$$
\begin{aligned}
& \overline{\tilde{H}}_{I S}^{(1)}=\frac{1}{2} H_{I S}^{Z Z}+\frac{1}{2} H_{I S}^{Y Y}-\frac{2}{\pi} H_{I S}^{Z Y}, \\
& \overline{\tilde{H}}_{I S}^{(2)}=\frac{1}{2} H_{I S}^{Y Y}+\frac{1}{2} H_{I S}^{X X}-\frac{2}{\pi} H_{I S}^{Y X}, \\
& \overline{\tilde{H}}_{I S}^{(3)}=\frac{1}{2} H_{I S}^{X X}+\frac{1}{2} H_{I S}^{Z Z}+\frac{2}{\pi} H_{I S}^{X Z}, \\
& \overline{\tilde{H}}_{I S}^{(4)}=\frac{1}{2} H_{I S}^{Z Z}+\frac{1}{2} H_{I S}^{Y Y}+\frac{2}{\pi} H_{I S}^{Z Y}, \\
& \overline{\tilde{H}}_{I S}^{(5)}=\frac{1}{2} H_{I S}^{Y Y}+\frac{1}{2} H_{I S}^{X X}+\frac{2}{\pi} H_{I S}^{Y X}, \\
& \overline{\tilde{H}}_{I S}^{(6)}=\frac{1}{2} H_{I S}^{X X}+\frac{1}{2} H_{I S}^{Z Z}-\frac{2}{\pi} H_{I S}^{X Z} .
\end{aligned}
$$

Analogous expressions in reverse order are obtained for the last six pulses: $\tilde{\tilde{H}}_{I S}^{(n)}=\overline{\tilde{H}}_{I S}^{(12-n+1)}, n=7, \ldots, 12$. In Eqs. (8), the notations $H_{I S}^{\alpha \alpha}=2 \omega_{I S} I_{\alpha} S_{\alpha}$ and $H_{I S}^{\alpha \beta}=\omega_{I S}\left(I_{\alpha} S_{\beta}+I_{\beta} S_{\alpha}\right)$, with $\alpha, \beta=X, Y, Z$, were employed. by

The average heteronuclear dipolar Hamiltonian is given

$$
\overline{\tilde{H}}_{I S}\left(\tau_{c}\right)=\frac{1}{3}\left[H_{I S}^{Z Z}+H_{I S}^{Y Y}+H_{I S}^{X X}\right]=\frac{2}{3} \omega_{I S} \mathbf{I} \cdot \mathbf{S} .
$$

Performing analogous calculation for the chemical shift contribution [see Eq. (2)], the spin Hamiltonian for the internal spin interactions is obtained in the form

$$
\tilde{\tilde{H}}_{\text {int }}\left(\tau_{c}\right)=\frac{2}{3} \omega_{I S} \mathbf{I} \cdot \mathbf{S}+\frac{2 \Omega_{I}}{3 \pi}\left[2 I_{x}+I_{z}\right]+\frac{2 \Omega_{S}}{3 \pi}\left[2 S_{x}+S_{z}\right] .
$$

With vanishing chemical shift terms, the spin Hamiltonian of Eq. (10) is invariant (isotropic) with respect to rotations of the axis frames. As inferred from the form of the last two terms, the direction of the effective rf fields is in the $x-z$ plane tilted at an angle $\alpha=\arctan (2) \approx 63.4^{\circ}$ away from the $z$ axis (Fig. 2).

It is convenient to express the Hamiltonian in the doubly tilted frame rotated through the angle $\alpha$ around the $y$ axis $\tilde{\tilde{H}}_{\text {int }}^{T}\left(\tau_{c}\right)=e^{i \alpha I_{y}+i \alpha S_{y}} \tilde{\tilde{H}}_{\text {int }}\left(\tau_{c}\right) e^{-i \alpha I_{y}-i \alpha S_{y}}$ :

$$
\tilde{\tilde{H}}_{\mathrm{int}}^{T}\left(\tau_{c}\right)=s_{I S} \omega_{I S} \mathbf{I} \cdot \mathbf{S}+s_{\mathrm{CS}} \Omega_{l} I_{z}+s_{\mathrm{CS}} \Omega_{S} S_{z},
$$

where $s_{\mathrm{CS}}=(2 \sqrt{ } 5) /(3 \pi) \approx 0.475$ is the chemical shift scaling factor of the BLEW12 sequence and $s_{I S}=2 / 3$ is the scaling factor of the heteronuclear spin interaction. Note that the scaling factors of chemical shift and dipolar coupling interactions are different in contrast to a single channel BLEW12 sequence, where the scaling of the heteronuclear coupling effective for the evolution of the single-quantum coherence coincides with that for chemical shift. ${ }^{9}$ The last two terms in the Hamiltonian [Eq. (11)] induce the precession of the magnetization component orthogonal to the effective rf field at the scaled chemical shift frequency. To avoid this effect, which may interfere with the dipolar coupling evolution, the spin magnetizations must be prepared along the $z$ axes of the tilted frames. Therefore, the tilt pulse in the pulse sequence given in Fig. 1(a) is necessary.

For further analysis it is convenient to rewrite the Hamiltonian [Eq. (11)] in terms of single transition operators in zero-quantum (ZQ) and double-quantum (DQ) subspaces, ${ }^{12,13}$

$\mathrm{ZQ}_{x}=I_{x} S_{x}+I_{y} S_{y}, \quad \mathrm{ZQ}_{y}=I_{y} S_{x}-I_{x} S_{y}, \quad \mathrm{ZQ}_{z}=\left(I_{z}-S_{z}\right) / 2$,

$\mathrm{DQ}_{x}=I_{x} S_{x}-I_{y} S_{y}, \quad \mathrm{DQ}_{y}=I_{y} S_{x}+I_{x} S_{y}, \quad \mathrm{DQ}_{z}=\left(I_{z}+S_{z}\right) / 2$

By keeping only relevant ZQ terms (zero-quantum, doublequantum, and longitudinal order operator mutually commute; moreover, DQ and longitudinal order operators do not affect the evolution if the initial density matrix is represented by a pure $\mathrm{ZQ}_{z}$ term), the Hamiltonian is expressed as

$$
\tilde{\tilde{H}}_{\mathrm{int}}^{T}=s_{I S} \omega_{I S} \mathrm{ZQ}_{x}+s_{\mathrm{CS}}\left(\Omega_{I}-\Omega_{S}\right) \mathrm{ZQ}_{z} .
$$

The transformation $\quad U_{\xi}=e^{i \xi \mathrm{ZQ}_{y}}, \quad \xi=\arctan \left[s_{\mathrm{CS}}\left(\Omega_{I}\right.\right.$ $\left.\left.-\Omega_{S}\right) / s_{\mathrm{CS}} \omega_{I S}\right]$, which corresponds to a rotation by an angle $\xi$ around the $y$ axis in the zero-quantum subspace, results in the spin Hamiltonian as shown below:

$$
U_{\xi}^{-1} \tilde{\tilde{H}}_{\mathrm{int}}^{T} U_{\xi}=\omega^{\mathrm{eff}} \mathrm{ZQ},
$$

where

$$
\omega_{I S}^{\text {eff }}=\frac{2}{3} \sqrt{\omega_{I S}^{2}+\frac{5}{\pi^{2}}\left(\Omega_{I}-\Omega_{S}\right)^{2}} .
$$

The time evolution of the density matrix (expressed in the tilted frame) is calculated as

$$
\sigma^{T}(t)=U_{\xi}\left[e^{i \omega^{\mathrm{eff}} \mathrm{ZQ}} \mathrm{Q}_{x}^{t}\left\{U_{\xi}^{-1} \sigma^{T}(0) U_{\xi}\right\} e^{-i \omega^{\mathrm{eff}} \mathrm{ZQ}_{x} t}\right] U_{\xi}^{-1} .
$$

Strictly, the time variable $t$ is a multiple of the rf cycle time $n \tau_{c}$.

An efficient heteronuclear spin exchange is obtained when the initial density matrix is prepared in a pure zero quantum state

$$
\sigma^{T}(0)=S_{z}-I_{z}=-2 Z Q_{z} .
$$

In this case, the time evolution of $\sigma^{T}(t)$ under the Hamiltonian [Eq. (14)] is given by

$$
\begin{aligned}
\sigma^{T}(t)= & -2\left(\cos ^{2} \xi \cos \omega_{I S}^{\mathrm{eff}} t+\sin ^{2} \xi\right) \mathrm{ZQ}_{z}-2\left(1-\cos \omega_{I S}^{\mathrm{eff}} t\right) \\
& \times \sin \xi \cos \xi \times \mathrm{ZQ}_{x}+2 \cos \xi \sin \omega_{I S}^{\mathrm{eff}} t \times \mathrm{ZQ}_{y} .
\end{aligned}
$$

The observed signal is $s(t) \propto \operatorname{Tr}\left\{\sigma^{T}(t) S_{z}\right\}$ (in the experiment, the signal is recorded following the tilt-back pulse to flip the magnetization to the transverse plane of the laboratory frame, see Figs. 1(a) and 2):

$$
s(t)=s(0)\left[\cos ^{2} \xi \cos \omega_{I S}^{\mathrm{eff}} t+\left(1-\cos ^{2} \xi\right)\right],
$$

where 


$$
\cos ^{2} \xi=\frac{\omega_{I S}^{2}}{\omega_{I S}^{2}+\left(5 / \pi^{2}\right)\left(\Omega_{I}-\Omega_{S}\right)^{2}} .
$$

In the frequency domain, this signal corresponds to a superposition of a doublet with a splitting of $2 \omega_{I S}^{\text {eff }}$ and a zero frequency peak. Thus, the effect of the resonance offset is the increasing dipolar splitting and the presence of the central peak in the spectrum. This is similar to the Lee-Goldburg spin-lock sequence employed in the polarization inversion and spin exchange at the magic angle (PISEMA) experiment. ${ }^{14}$ For on-resonance condition the spectrum consists of a doublet with a splitting of $4 / 3 \omega_{I S}$.

\section{BLEW48 spin lock}

The longer homonuclear decoupling sequence BLEW48 is more tolerant to $\mathrm{rf}$ error terms such as those resulting from rf inhomogeneity and pulse length misadjustment. ${ }^{9}$ Since the effective rf field of this sequence is in the transverse plane (along the $-y$ direction), the experiment does not require a tilt pulse. The spin Hamiltonian of the BLEW48 spin-lock irradiation is calculated analogously to the previous sequence as presented above. When expressed in the frame rotated by $90^{\circ}$ around the $x$ axis, so that the $z$ axis is along the effective field of the BLEW48 sequence, it takes the form

$$
\overline{\tilde{H}}_{\mathrm{int}}^{T}\left(\tau_{c}\right)=\frac{2}{3} \omega_{I S} \mathbf{I} \cdot \mathbf{S}-\frac{4}{3 \pi} \Omega_{I} I_{z}-\frac{4}{3 \pi} \Omega_{S} S_{z} .
$$

Here, the chemical shift scaling factor $s_{\mathrm{CS}}=4 /(3 \pi) \approx 0.424$ is somewhat lower compared to the BLEW12 sequence, while the scaling factor of the heteronuclear spin interaction $s_{I S}$ $=2 / 3$ coincides with that of the BLEW12 spin-lock scheme. The observed signal is given by an expression analogous to Eq. (19) with

$$
\omega_{I S}^{\text {eff }}=\frac{2}{3} \sqrt{\omega_{I S}^{2}+\frac{4}{\pi^{2}}\left(\Omega_{S}-\Omega_{I}\right)^{2}}
$$

and

$$
\cos ^{2} \xi=\frac{\omega_{I S}^{2}}{\omega_{I S}^{2}+\left(4 / \pi^{2}\right)\left(\Omega_{S}-\Omega_{I}\right)^{2}} .
$$

Figure 3 shows the frequency offset dependence of the oscillation frequency $\omega_{I S}^{\text {eff }}$ calculated using Eqs. (15) and (22). Its value is normalized to the value at zero offset $s_{I S} \omega_{I S}$, while the offset scale is given in the units of the dipolar coupling constant $\omega_{I S}$. For comparison, the analogous dependence for the Lee-Goldburg (LG) spin lock of the PISEMA experiment ${ }^{6}$ is included. Clearly, for all these experiments the effect is significant for the offset values comparable to the magnitude of the dipolar coupling and leads to large errors in the measurement of small coupling constants.

\section{On-resonance BLEWn spin lock}

The isotropic form of the dipolar coupling term in the Hamiltonians [Eqs. (11) and (21)] suggests that, at exact resonance conditions, in BLEWn spin-lock experiments, an arbitrary magnetization component of one spin can be transferred to its counterpart of another spin. For example, with

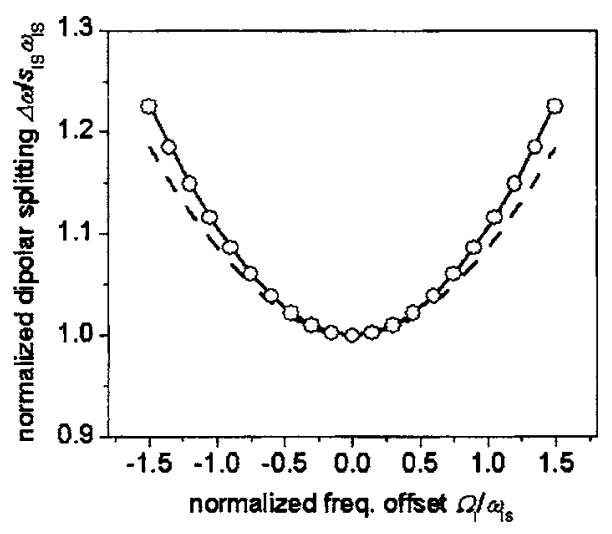

FIG. 3. Frequency offset dependence, $\omega_{I S}^{\text {eff }}\left(\Omega_{l}\right)$ (at $\left.\Omega_{S}=0\right)$, of the oscillation frequency for the HIMSELF sequence based on BLEW12 (solid line), BLEW48 (dashed line), and Lee-Goldburg (circles) homonuclear decoupling sequences. The vertical scale is normalized to the value of $s_{I S} \omega_{I S}$, while the offset scale is given in the units of the dipolar coupling constant $\omega_{I S}$.

the initial density matrix, $\sigma(0)=S_{z}-I_{z}$, corresponding to $z$ magnetizations in the laboratory frame, the time evolution of the density matrix is obtained in the form

$$
\sigma(t)=2 \mathrm{ZQ}_{z} \cos \frac{2}{3} \omega_{I S} t-2 \mathrm{ZQ}_{y} \sin \frac{2}{3} \omega_{I S} t,
$$

where $t=n \tau_{c}$. The signal detected in the $S$ spin channel (after applying the $90^{\circ}$ pulse to flip the magnetization in the transverse plane) is

$$
s(t) \propto \cos \frac{2}{3} \omega_{I S} t .
$$

Analogously, the same signals are obtained for the initial density matrices $\sigma(0) \propto S_{x}-I_{x}$ and $\sigma(0) \propto S_{y}-I_{y}$.

\section{B. Broadband BLEWn spin-lock sequences}

The performance of the BLEWn spin-lock sequence with respect to suppressing the frequency offset effects is improved by extending the sequence with a pair of $180^{\circ}$ pulses applied simultaneously in both rf channels at the end of a rf cycle as shown in Fig. 1(b). When applied at a rate faster than the frequency offset and the dipolar coupling values, these pulses refocus the effects of $S$ and $I$ spin evolutions under the chemical shift/frequency offset, while the heteronuclear spin interaction, described by the bilinear spin operator, remains intact. Due to noncommutation of the dipolar and chemical shift terms in the Hamiltonian, the refocusing of the chemical shift evolution must be performed at sufficiently short time intervals so that no significant polarization exchange takes place between the refocusing pulses (see below).

\section{BB-BLEW12 spin lock}

In the offset refocused BLEW12 spin-lock sequence, $180^{\circ}$ pulses of length $\tau_{180}$ are applied along the $y$ direction in the rotating frame, i.e., perpendicular to the effective field direction of the BLEW12 irradiation, as shown in Fig. 2. The rf cycle for each frequency channel is given by 


$$
\left(180^{0}\right)_{y}-\mathrm{BLEW} 12-\left(180^{\circ}\right)_{y}-\mathrm{BLEW} 12 .
$$

The cycle time is $\tau_{c}=2 \tau_{180}+24 \tau_{90}$. Using Eqs. (6) and (7), the zeroth-order average heteronuclear spin Hamiltonian is calculated to be

$$
\begin{aligned}
\overline{\tilde{H}}_{\mathrm{int}}\left(\tau_{c}\right)= & \frac{2}{3} \frac{\omega_{I S}}{\tau_{c}}\left[3 \tau_{180}\left(I_{z} S_{z}+I_{x} S_{x}\right)\right. \\
& \left.+24 \tau_{90}\left(I_{z} S_{z}+I_{y} S_{y}+I_{x} S_{x}\right)\right] .
\end{aligned}
$$

As expected, the Zeeman terms (chemical shift and frequency offset) vanish in the average Hamiltonian. With the use of infinitely short $180^{\circ}$ refocusing pulses, but finite $90^{\circ}$ BLEW12 pulses, the Hamiltonian in Eq. (27) converges to the isotropic form $\overline{\tilde{H}}_{\text {int }}\left(\tau_{c}\right)=(2 / 3) \omega_{I S} \mathbf{I} \cdot \mathbf{S}$. At a finite $180^{\circ}$ pulse length, the Hamiltonian is not completely isotropic. While the last term in Eq. (27) preserves the threedimensional (3D) isotropy, the first term is invariant to the rotation only about the $y$ axis. Hence, in accordance with the symmetry of the Hamiltonian, two kinds of initial conditions are distinguished: with the initial density matrix corresponding to the magnetization in the $x z$ plane or along the $y$ axis. Depending on the selection of initial conditions, different terms in the dipolar coupling Hamiltonian contribute to the time evolution of the density matrix.

Initial magnetization in the $x z$ plane. We arbitrarily chose $S$ and $I$ spin magnetizations prepared along the $z$ axis in the doubly tilted double rotating frame. In this case, using the definitions of Eq. (12) and, as before, neglecting the DQ and longitudinal order terms, Eq. (27) transforms to

$$
\overline{\tilde{H}}_{I S}\left(\tau_{c}\right)=s_{z x} \omega_{I S} \mathrm{ZQ}_{x},
$$

where

$$
s_{z x}=\frac{2}{3}\left(1-\frac{\tau_{180}}{2 \tau_{c}}\right)
$$

is the heteronuclear dipolar scaling factor.

Initial magnetization along the $y$ axis. In this situation, it is convenient to rotate the axis frame by $90^{\circ}$ around the $x$ axis, so that the Hamiltonian in Eq. (27) becomes

$$
\overline{\tilde{H}}_{I S}\left(\tau_{c}\right)=s_{y} \omega_{I S} \mathrm{ZQ},
$$

where the scaling factor is given by

$$
s_{y}=\frac{2}{3}\left(1+\frac{\tau_{180}}{\tau_{c}}\right) .
$$

Thus, the dipolar coupling scaling factors and hence the frequencies of the dipolar coupling oscillation during the spin exchange in the broadband BLEW12 (BB-BLEW12) spinlock sequence depend on the selection of the initial magnetization. For example, with $\tau_{180}=2 \tau_{90}$, the scaling factors $s_{z x}=0.643$ and $s_{y}=0.714$ are, respectively, slightly lower and higher compared to a nonrefocused sequence, where $s=0.667$.

While, the mixing Hamiltonian [Eq. (27)] is not strictly isotropic, it provides an efficient transfer of any magnetization component. The frequency of the polarization exchange, however, varies according to the scaling factors of Eqs. (29) and (31). In general, if the initial magnetization has components both along the $y$ axis and in the $x z$ plane, two doublets in the dipolar coupling spectrum result with the splittings of $2 s_{y} \omega_{I S}$ and $2 s_{x z} \omega_{I S}$, respectively.

\section{BB-BLEW48 spin lock}

In the refocused BLEW48 spin-lock sequence, the rf field during the $180^{\circ}$ pulses are applied along the $x$ axis of the rotating frame, i.e., perpendicular to the effective field of the BLEW48 irradiation. The rf cycle is given by

$$
\left(180^{\circ}\right)_{x}-\mathrm{BLEW} 48-\left(180^{\circ}\right)_{x}-\mathrm{BLEW} 48
$$

with the cycle time of $\tau_{c}=2 \tau_{180}+96 \tau_{90}$. Using Eqs. (6) and (7), the zeroth-order average heteronuclear spin Hamiltonian is calculated to be

$$
\begin{aligned}
\overline{\tilde{H}}_{\text {int }}\left(\tau_{c}\right)= & \frac{2}{3} \frac{\omega_{I S}}{\tau_{c}}\left[3 \tau_{180}\left(I_{z} S_{z}+I_{y} S_{y}\right)\right. \\
& \left.+96 \tau_{90}\left(I_{z} S_{z}+I_{y} S_{y}+I_{x} S_{x}\right)\right] .
\end{aligned}
$$

Similar to the BLEW12-based sequence, the last term in Eq. (23) preserves the $3 \mathrm{D}$ isotropy, while the first term is invariant to the rotation only around the $x$ axis.

Initial magnetization in the $y z$ plane. In this case, by keeping only those terms that lead to the polarization exchange, the Hamiltonian given in Eq. (33) reduces to

$$
\overline{\tilde{H}}_{I S}\left(\tau_{c}\right)=s_{z y} \omega_{I S} \mathrm{ZQ} x
$$

with a scaling factor

$$
s_{z y}=\frac{2}{3}\left(1-\frac{\tau_{180}}{2 \tau_{c}}\right) \text {. }
$$

Initial magnetization along the $x$ axis. By performing a $90^{\circ}$ rotation around the $x$ axis, the Hamiltonian [Eq. (33)] becomes

$$
\overline{\tilde{H}}_{I S}\left(\tau_{c}\right)=s_{x} \omega_{I S} \mathrm{ZQ},
$$

where the scaling factor is given by

$$
s_{x}=\frac{2}{3}\left(1+\frac{\tau_{180}}{\tau_{c}}\right) .
$$

When the same rf power level is used for BLEW pulses and $180^{\circ}$ pulses, i.e., $\tau_{180}=2 \tau_{90}$, the scaling factors are $s_{z x}=0.660$ and $s_{y}=0.680$. It is also possible to apply $180^{\circ}$ pulses after half the duration of the BLEW 48 cycle, which leads to $s_{z x}=0.654$ and $s_{y}=0.690$.

While BLEW48 is superior to BLEW12 in terms of the tolerance to rf errors, it has a disadvantage of long rf cycle time which limits the spectral width for the observation. In the refocused sequences, the rf cycle time is further increased by a factor of about 2 . It is, however, possible to sample twice during the full cycle, that is, after each BLEW48-180 block. If magnetization is initially in the $z y$ plane, one needs to invert the receiver phase in subsequent increment points since magnetization is inverted by the $180_{ \pm x}^{\circ}$ pulse. That is not required, however, if the magnetization is prepared along the $x$ axis. Furthermore, sampling after half the BLEW48 cycle is also possible, as well as inserting the refocusing 


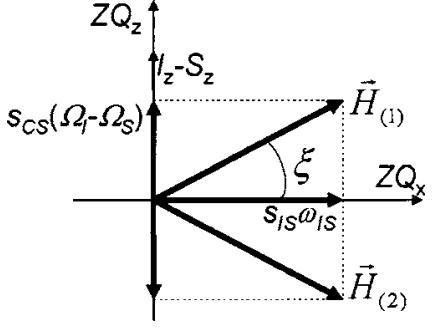

FIG. 4. Schematic representation of the direction of the effective Hamiltonian in the zero-quantum subspace. Initial magnetization $\sigma(0)=I_{z}-S_{z}$ is along the $z$ axis and processes around the direction of the effective Hamiltonian, $H$.

pulses. Our experimental results suggest that the use of the $\left(180^{\circ}-\overline{180^{\circ}}-\overline{180^{\circ}}-180^{\circ}\right)$ phase cycling ${ }^{15}$ improves the spectral shape in the presence of the rf field inhomogeneity.

\section{Dependence on the time interval between $180^{\circ}$ pulses}

In the calculation above it was assumed that the intervals between $180^{\circ}$ pulses are much shorter compared to the period of dipolar oscillation. This ensured that no significant polarization exchange takes place between refocusing pulses, and therefore, an efficient suppression of the chemical shift/ frequency offset terms is guaranteed. However, at longer intervals $\tau \sim 1 / \omega_{I S}$, due to noncommutation of the dipolar and chemical shift terms in the spin Hamiltonian, the refocusing is not complete. This effect can most straightforwardly be appreciated by considering the schematic representation of the direction of the effective Hamiltonian in the zeroquantum subspace. It is assumed here that the $180^{\circ}$ pulse width is infinitely short, while the rf field strength during the BLEW irradiation has a finite value. The direction of the effective Hamiltonian in the presence of the resonance offset, which defines the precession axis, is tilted at an angle $\xi=\arctan \left[s_{\mathrm{CS}}\left(\Omega_{I}-\Omega_{S}\right) /\left(s_{I S} \omega_{I S}\right)\right]$ as shown in the vector diagram in Fig. 4. Following the pair of $180^{\circ}$ pulses the precession axis flips, while the precession frequency $\omega_{I S}^{\text {eff }}$ remains the same. An analogous vector representation has been used for describing the Hartmann-Hahn mismatch effect in the flip-flop LG sequence of PISEMA. ${ }^{16}$

During the interval $\tau$ between the $180^{\circ}$ pulses, the magnetization rotates through an angle

$$
\begin{aligned}
\beta_{1}(\tau)=\omega^{\text {eff }} \tau & =\sqrt{s_{\mathrm{CS}}^{2}\left(\Omega_{I}-\Omega_{S}\right)^{2}+s_{I S}^{2} \omega_{I S}^{2}} \times \tau \\
& =\sqrt{\tan ^{2} \xi+1} \times \beta_{1}^{(0)}(\tau),
\end{aligned}
$$

where $\beta_{1}^{(0)}(\tau)=s_{I S} \omega_{I S} \tau$ is the angle of rotation in the absence of resonance offset. In the theory of composite pulses, ${ }^{17}$ it is shown that two consecutive noncommuting rotations through the same angle $\beta_{1}$ about alternating axes as shown in Fig. 4 correspond to a single rotation about an effective axis in the $x-y$ plane through an angle

$$
\cos \frac{\beta_{12}}{2}=\cos ^{2} \frac{\beta_{1}}{2}-\sin ^{2} \frac{\beta_{1}}{2}\left(\cos ^{2} \xi-\sin ^{2} \xi\right) .
$$

The apparent angular velocity is $\omega_{I S}^{\mathrm{app}}=\beta_{12} / 2 \tau$. Figure 5(a) displays the frequency $\omega_{I S}^{\mathrm{app}}$, normalized to the value of the oscillation frequency at zero offset $\omega_{I S}^{(0)}=s_{I S} \omega_{I S}$, as a function

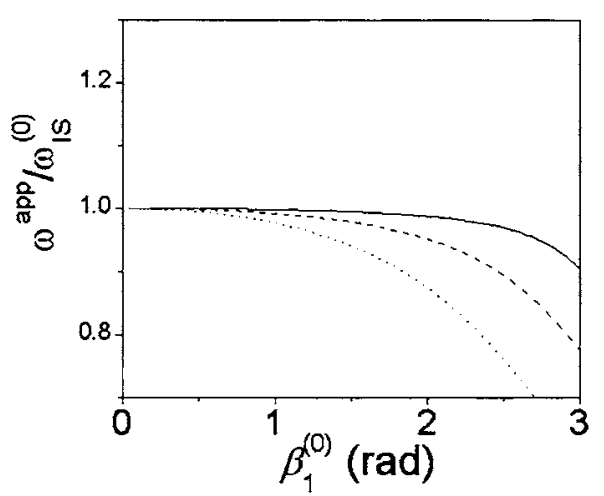

(a)

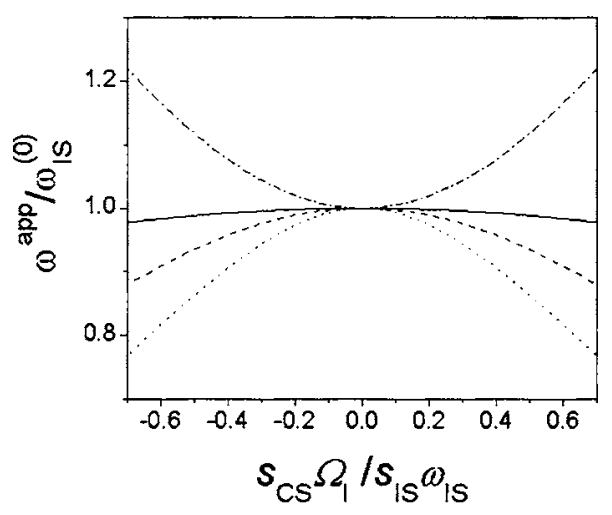

(b)

FIG. 5. (a) Apparent oscillation frequency $\omega_{I S}^{\text {app }}$, normalized to the value of the frequency at zero offset $\omega_{I S}^{(0)}=s_{I S} \omega_{I S}$, as a function of the angle $\beta_{1}^{(0)}(\tau)$ $=\omega_{I S}^{(0)} \tau$. The solid, dashed, and dotted curves are calculated for different ratios of the chemical shift to dipolar coupling constants $\Omega_{l} / \omega_{I S}=0.3,0.6$, and 1.0, respectively (with $\Omega_{S}=0$ ). (b) Dependence of the normalized oscillation frequency $\omega_{I S}^{\mathrm{app}} / \omega_{I S}^{(0)}$ on the scaled chemical shift $s_{\mathrm{CS}} \Omega_{l}$ normalized to the scaled dipolar coupling constant $s_{I S} \omega_{I S}$. The solid, dashed, and dotted curves are calculated for different angles $\beta_{1}^{(0)}(\tau)=\omega_{I S}^{(0)} \tau=1.0,2.0$, and 2.5, radian respectively. The data for nonrefocused sequence BLEW12 spin lock is also included (dash-dot line).

of the angle $\beta_{1}^{(0)}(\tau)=\omega_{I S}^{(0)} \tau$. The curves are shown for different ratios of the chemical shift to dipolar coupling constant $\Omega_{I} / \omega_{I S}\left(\right.$ at $\left.\Omega_{S}=0\right)$. It is seen that the chemical shift effect is suppressed if the angle $\beta_{1}^{(0)}(\tau)$ through which the magnetization rotates during the interval $\tau$ between refocusing pulses is sufficiently small. Also, suppressing the larger chemical shift requires correspondingly smaller angle $\beta_{1}^{(0)}(\tau)$, i.e., shorter delays $\tau$ for a given dipolar coupling constant. For instance, for $\mathrm{rf}$ field $\gamma B_{1} / 2 \pi=100 \mathrm{kHz}$ during BLEW12, the delay between $180^{\circ}$ pulses (assuming infinitely short pulses after each BLEW12 cycle) is $\tau=12 \times 2.5(\mu \mathrm{s})=30 \mu \mathrm{s}$. With the dipolar coupling constant of $\omega_{I S} / 2 \pi=10 \mathrm{kHz}$, the angle of rotation is $\beta_{1}^{(0)}=s_{I S} \omega_{I S} \tau \approx 1.26 \mathrm{rad}$. Hence, as can be seen in Fig. 5(a), the effect of frequency offset up to $10 \mathrm{kHz}$ will be suppressed within an error of less than $4 \%$.

Data in Fig. 5(b) show the offset dependence of the normalized oscillation frequency $\omega_{I S}^{\text {app }} / \omega_{I S}^{(0)}$ for different angles $\beta_{1}^{(0)}(\tau)=\omega_{I S}^{(0)} \tau$. The smaller the angle $\beta_{1}^{(0)}$ (i.e., a shorter interval $\tau$ ), the wider the range of the efficient suppression of the chemical shift effects. The offset effect of the oscillation frequency in the nonrefocused BLEW12 spin-lock sequence is also shown for comparison. 


\section{WIM24 sequence}

The WIM24 sequence has been specifically developed for the heteronuclear polarization transfer in heteronuclear chemical shift correlation (HETCOR) experiments. ${ }^{10}$ It has been designed using the same principles as the BLEWn technique and has similar homonuclear decoupling performance. However, the chemical shift evolution is deliberately not preserved in WIM, in contrast to BLEWn.

The cyclic properties of the WIM24 sequence are as follows: (i) the chemical shift term is refocused during the block of six $90^{\circ}$ pulses: (ii) in the heteronuclear dipolar Hamiltonian the cross terms of the type $H_{I S}^{\alpha \beta}$, see Eqs. (8), vanish after $1290^{\circ}$ pulses. The completion of the 24 pulses in the WIM sequence is required merely to suppress the $\mathrm{rf}$ pulse errors. Further compensation of the rf error, such as missetting of $90^{\circ}$ pulse length and mismatch of the rf fields in the two channels, is achieved by phase cycling, ${ }^{15}$ WIM24-WIM24-WIM24-WIM24. This cycle also improves the line shape in the presence of a large frequency offset.

For the $I-S$ spin pair the zeroth-order average Hamiltonian calculated according to Eqs. (4)-(7) takes the isotropic mixing form

$$
\overline{\tilde{H}}_{\mathrm{int}}^{T}\left(\tau_{c}\right)=\frac{2}{3} \omega_{I S} \mathbf{I} \cdot \mathbf{S}
$$

In contrast to the BLEW-CP sequences, the average WIM Hamiltonian preserves the isotropic form in the presence of resonance offset/chemical shift and thus can be used for the polarization transfer of arbitrary magnetization component with equal efficiency. Practically, the experiment involving the $z$-component exchange is most tolerant to rf errors and chemical shift offsets at the limited power level as is shown below by numerical simulation.

\section{EXPERIMENT}

The performance of the HIMSELF sequence was experimentally demonstrated on samples of ${ }^{15} \mathrm{~N}$-labeled $n$-acetyl-L${ }^{15} \mathrm{~N}$-valyl-L- ${ }^{15} \mathrm{~N}$-leucine (NAVL) single crystal and the thermotropic discotic liquid crystal hexahexyloxy-triphenylene (THE6) with the natural isotopic abundance. THE6 forms a hexagonal columnar mesophase, where the molecules arranged in the columns undergo fast rotation around the molecular symmetry axis. ${ }^{18}$ Experiments were performed on a stationary sample in the mesophase at a temperature of $85^{\circ} \mathrm{C}$. Prior to the experiment, the sample was macroscopically oriented with the columnar axes perpendicular to the magnetic field direction of the spectrometer by slow cooling from the isotropic phase.

All NMR experiments were performed at a magnetic field of $9.4 \mathrm{~T}$ on a Chemagnetics/Varian Infinity-400 spectrometer equipped with a commercial $5 \mathrm{~mm}$ doubleresonance magic angle spinning (MAS) probe (used in static mode) and on a home-built static probe at the University of Michigan. Numerical simulations were performed using the SIMPSON platform at the University of Michigan. ${ }^{19}$
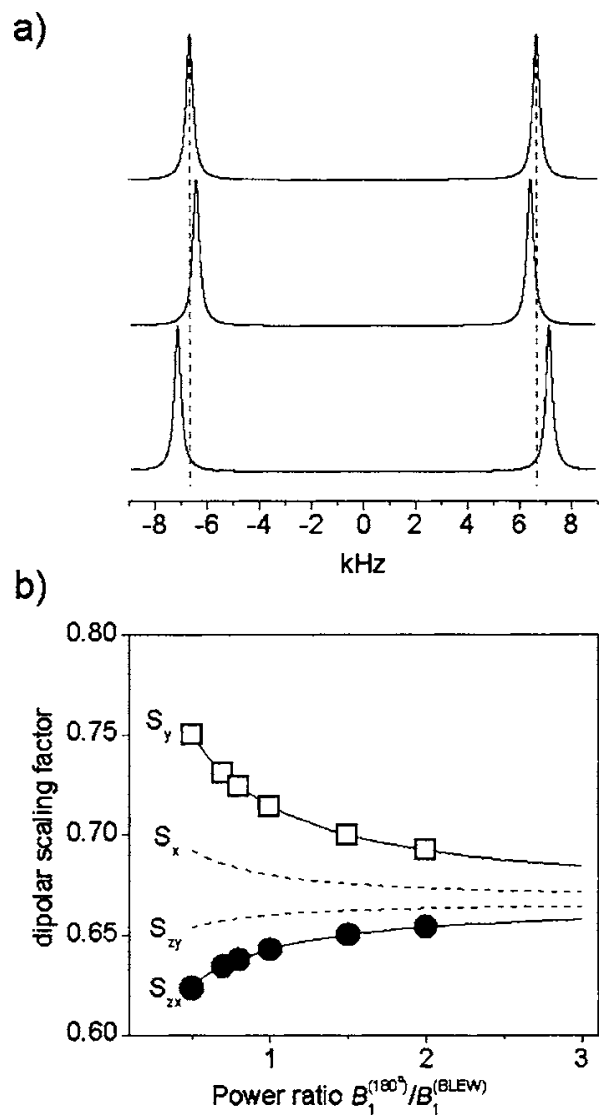

FIG. 6. (a) Numerically calculated dipolar coupling spectra for the $\mathrm{CH}$ spin pair with a coupling constant $d_{I S}=\omega_{I S} / 2 \pi=10 \mathrm{kHz}$ for BLEW12-based experiments at on-resonance conditions. $\gamma B_{1} / 2 \pi=62.5 \mathrm{kHz}$ and line broadening of $300 \mathrm{~Hz}$ were used in the simulations. Top spectrum: BLEW12 spin lock; middle and bottom spectra: BB-BLEW12 spin lock with the initial magnetizations along the $z$ and $y$ directions, respectively. rf field strength for $180^{\circ}$ pulses was $62.5 \mathrm{kHz}$. (b) The dependence of the dipolar coupling scaling factor on the ratio of the rf power of the $180^{\circ}$ pulse to that of BLEW irradiation. Symbols: SIMPSON simulations for BB-BLEW12 spin lock with $\omega_{I S} / 2 \pi=10 \mathrm{kHz}$ and $\mathrm{rf}$ field during the BLEW irradiation $\gamma B_{1} / 2 \pi$ $=62.5 \mathrm{kHz}$ for the initial conditions $\sigma(0)=-I_{z}+S_{z}$ (circles) and $\sigma(0)=-I_{y}$ $+S_{y}$ (squares). The lines $s_{z x}$ and $s_{y}$ (BB-BLEW12 spin lock) and $s_{z y}$ and $s_{x}$ (BB-BLEW48 spin lock) were analytically calculated from the zeroth-order average Hamiltonian.

\section{SIMULATIONS}

\section{A. IS spin pair \\ 1. Scaling factor of the BB-BLEWn spin-lock sequences}

Analytical expressions for the dipolar coupling scaling factors of the refocused BLEWn spin-lock sequences obtained using the zeroth-order average Hamiltonian theory are applicable in the limit of the infinitely strong rf fields. The results of the numerical simulations for the practical rf power and dipolar coupling values are shown in Fig. 6. Calculation of the dipolar coupling spectra for the $\mathrm{CH}$ spin pair at exact resonance conditions (frequency offset effects are discussed in the next subsection) is presented for BLEW12-based experiments [Fig. 6(a)]. The effect of the refocusing pulses on the spectral shape is merely a small change of the spectral splitting, which also depends on the initial condition. Smaller and larger splittings, compared to the conventional BLEW12, are obtained for the exchange of $z$ and $y$ compo- 
a)

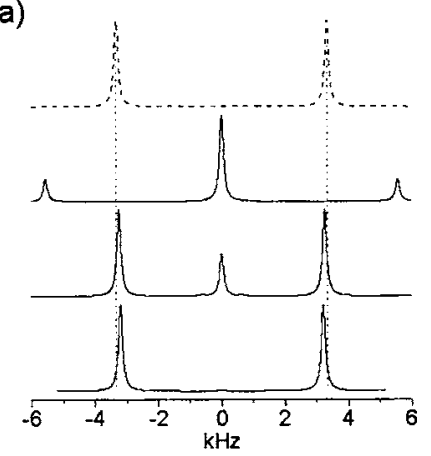

b)

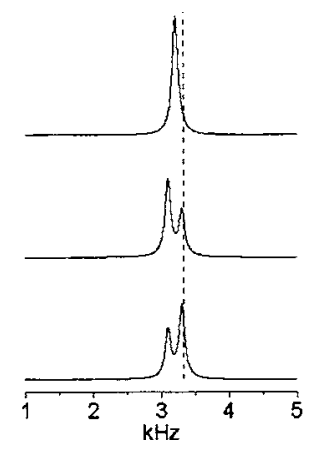

FIG. 7. Numerically calculated dipolar coupling spectra for the $\mathrm{CH}$ spin pair with $\omega_{I S} / 2 \pi=5 \mathrm{kHz}$ and $\gamma B_{1} / 2 \pi=62.5 \mathrm{kHz}$. (a) Top two spectra were obtained using BLEW12 spin lock at on-resonance (dashed) and with the ${ }^{1} \mathrm{H}$ offset of $10 \mathrm{kHz}$. (solid). Initial condition was defined by the density matrix $\sigma(0)=\left[-\left(2 I_{x}-I_{z}\right)+\left(2 S_{x}-S_{z}\right)\right] / 5^{1 / 2}$. The third spectrum from the top and bottom-most spectrum were, respectively, obtained using BB-BLEW12 spin-lock and WIM24 sequences at a ${ }^{1} \mathrm{H}$ offset frequency of $10 \mathrm{kHz}$ and with the initial density matrices $\sigma(0)=-I_{y}+S_{y}$ and $\sigma(0)=-I_{z}+S_{z}$. A line broadening of $300 \mathrm{~Hz}$ was used in processing the spectra. (b) Comparison of the line shapes in WIMz (top), WIM $x$ (middle), and WIM $y$ (bottom) dipolar spectra. Only the positive frequency peak of the dipolar split doublet is shown. A proton offset frequency of $10 \mathrm{kHz}$ and a line broadening of $100 \mathrm{~Hz}$ were used. The dotted line indicates the line position in the absence of the frequency offset.

nents, respectively. The dependence of the dipolar coupling scaling factor on the ratio of the rf power of the $180^{\circ}$ pulse to that of BLEW irradiation, $B_{1}^{\left(180^{\circ}\right)} / B_{1}^{(\mathrm{BLEW})}$, was calculated using numerical simulation. It compares well to the prediction derived from the average Hamiltonian theory [Fig. 6(b)]. As compared to the BB-BLEW12 spin lock, the scaling factors for the BB-BLEW48 spin-lock sequence are closer to the nominal value $s_{I S}=2 / 3$, but the intervals between $180^{\circ}$ pulses are longer so that the offset refocusing may not be efficient.

\section{Frequency offset}

Due to the presence of a Zeeman-type term in the zerothorder average BLEWn spin-lock Hamiltonian, the polarization exchange frequency is strongly affected by the resonance offset, as demonstrated for the BLEW12 spin-lock sequence in Fig. 7(a). Notice that not only the dipolar splitting changes in the presence of the offset, but also the intensity of peaks in the doublet significantly decreases while the intensity of the noninformative zero-frequency peak increases. In the refocused sequence as well as in WIM24, the offset effect is suppressed. It should be noted that the spectra obtained with the WIM24 scheme involving the exchange of $x$ or $y$ magnetization components may exhibit fine splitting in the presence of a large offset [Fig. 7(b)]. This is not observed for the case of the $z$ component exchange in the WIM $z$ sequence.

The simulated dipolar frequency values are plotted in Fig. 8 as a function of the offset frequency. Spin-locking using the BLEW12 sequence results in a strong offset frequency effect which increases dramatically when the dipolar coupling constant decreases, ca. Figs. 8(a) and 8(b). The WIM sequence is more efficient in suppressing this effect as compared to the BB-BLEW12 spin lock. The identical curves are obtained for ${ }^{1} \mathrm{H}$ and ${ }^{13} \mathrm{C}$ offsets. For the BB-BLEW48 spin-lock sequence the distance between $180^{\circ}$ pulses, which where inserted after each 24 pulses, is not sufficiently short to suppress the offset effect in the case of a larger dipolar coupling of $\omega_{I S} / 2 \pi=10 \mathrm{kHz}$, as shown in Fig. 8(c).

\section{3. rf errors}

Impact of rf errors on the spectral splittings was examined assuming on-resonance conditions with the dipolar coupling and $B_{1}$ field values of 10 and $100 \mathrm{kHz}$, respectively. The data shown in Fig. 9 are normalized to the splitting at ideal rf fields. Two kinds of rf errors were considered: missetting of the $90^{\circ}$ pulse length and mismatch of the ${ }^{1} \mathrm{H}$ and ${ }^{13} \mathrm{C} B_{1}$ field strengths. As expected, ${ }^{9}$ the BLEW48 sequence was much better in compensating for the missetting of the $90^{\circ}$ pulse length compared to BLEW12; however, it has the disadvantage of strong sensitivity to mismatch of the field in two frequency channels. Broadband BLEW sequences are, in addition to greater performance with respect to the frequency offset, also better in compensating for rf mismatch. (a)

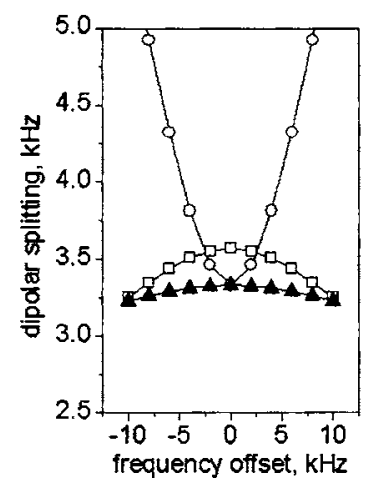

(b)

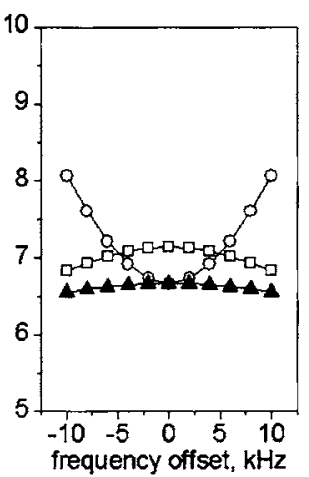

(c)

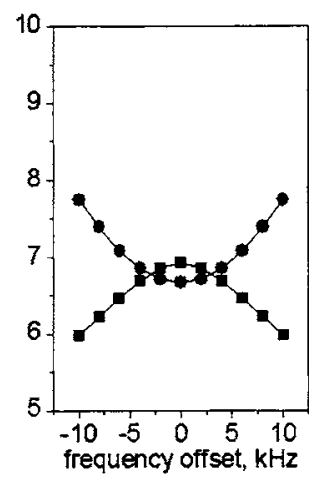

FIG. 8. The numerically simulated dipolar frequency as a function of the proton frequency offset: (a) $\omega_{I S} / 2 \pi$ $=5 \mathrm{kHz}, \quad \gamma B_{1} / 2 \pi=62.5 \mathrm{kHz} ; \quad$ (b) $\omega_{I S} / 2 \pi=10 \mathrm{kHz}$, $\gamma B_{1} / 2 \pi=100 \mathrm{kHz}$. (A higher $B_{1}$ field was used to increase the spectral window when simulating spectra with $\omega_{I S} / 2 \pi=10 \mathrm{kHz}$.) Circles: BLEW12 spin lock with $\sigma(0)=\left[-\left(2 I_{x}-I_{z}\right)+\left(2 S_{x}-S_{z}\right)\right] / 5^{1 / 2}$, squares: BB-BLEW12 spin lock with $\sigma(0)=-I_{y}+S_{y}$, triangles: WIM24 with $\sigma(0)=-I_{z}+S_{z}$, (c) Circles: BLEW48/2 spin lock $\left[\sigma(0)=-I_{y}+S_{y}\right]$, squares: BB-BLEW48/2 spin lock $\left[\sigma(0)=-I_{x}+S_{x}\right] ; \omega_{I S} / 2 \pi=10 \mathrm{kHz}, \gamma B_{1} / 2 \pi$ $=100 \mathrm{kHz}$. 

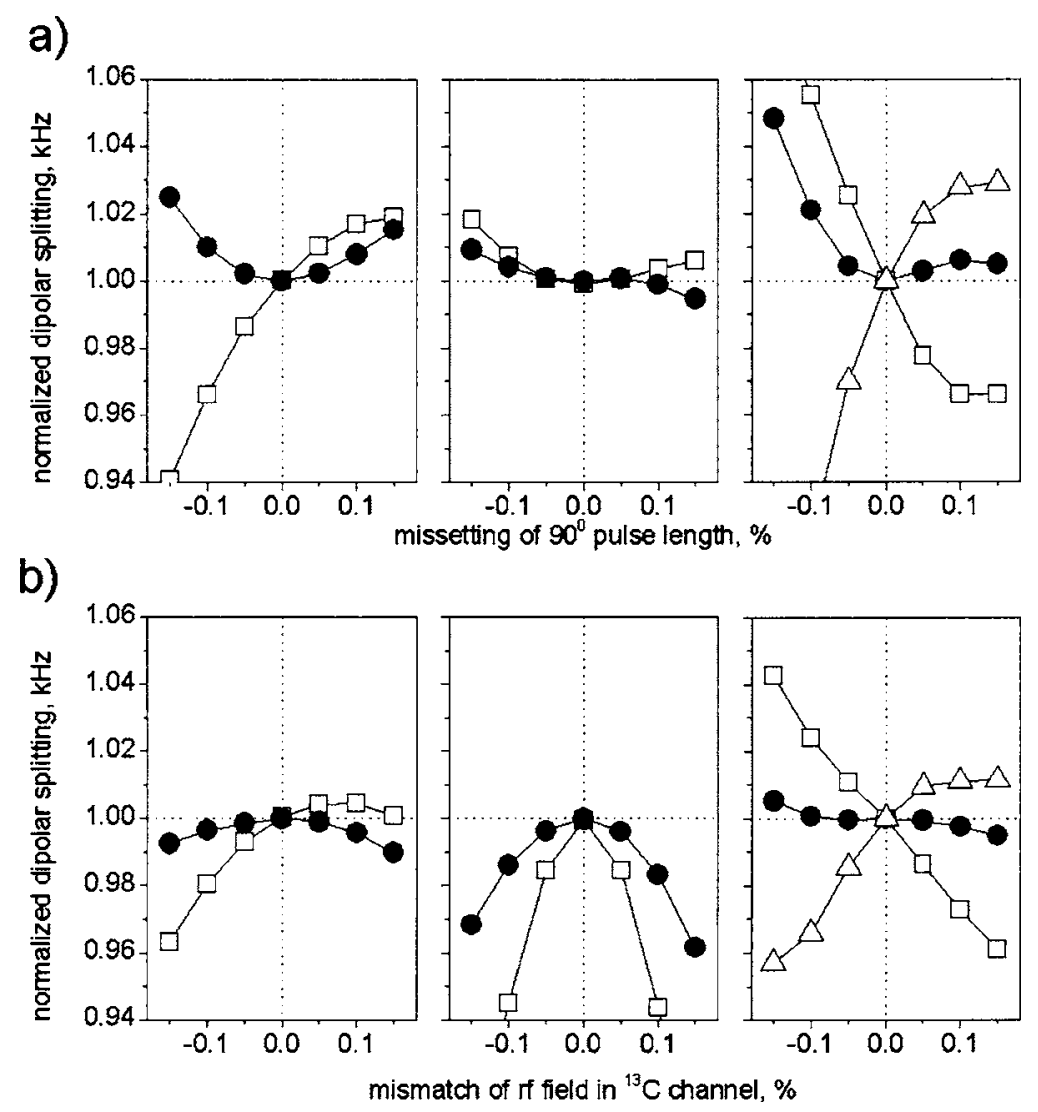

FIG. 9. Numerical simulation of the effects on the dipolar splittings of (a) missetting the $90^{\circ}$ pulse length and (b) mismatch of the ${ }^{1} \mathrm{H}$ and ${ }^{13} \mathrm{C} B_{1}$ field strengths. Left column: BLEW12 spin lock (squares) and BBBLEW12 spin lock (circles), middle column: BLEW48 spin lock (squares) and BB-BLEW48/2 spin lock (circles), right column: WIMz (circles), WIMy (squares), and WIM $x$ (triangles). On-resonance conditions are assumed and the dipolar coupling and $B_{1}$ field values are 10 and $100 \mathrm{kHz}$, respectively. The data are normalized to the splitting at ideal rf fields.

Splitting obtained with WIM $z$ is exceptionally tolerant to $B_{1}$ mismatch, while underestimation of the $90^{\circ}$ pulse length may result in a large error. It is also obvious that WIM sequences involving $x$ or $y$ component exchange are less robust to the rf errors compared to WIM with the $z$ component transfer. A frequency offset up to $10 \mathrm{kHz}$ did not alter significantly the results.

\section{4. $\mathrm{SI}_{2}$}

The calculated spectra of a mobile methylene $\mathrm{CH}_{2}$ group with the equivalent residual coupling $d_{\mathrm{CH}(1)}=d_{\mathrm{CH}(2)}$ are shown in Fig. 10. An important case often met in practice is

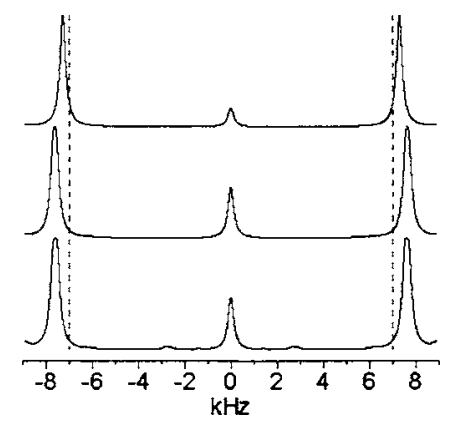

FIG. 10. Numerically calculated BB-BLEW12 spin-lock dipolar spectra of the fast rotating methylene $\mathrm{CH}_{2}$ group. A heteronuclear dipolar coupling constant $d_{\mathrm{CH}(1)}=d_{\mathrm{CH}(2)}=7 \mathrm{kHz}$ and $B_{1}$ field of $62.5 \mathrm{kHz}$ were used with a line broadening of $300 \mathrm{~Hz}$. The top spectrum was obtained at a zerofrequency offset and a homonuclear proton coupling $d_{\mathrm{HH}}=0$, while the middle spectrum was obtained using a zero-frequency offset and $d_{\mathrm{HH}}$ $=10 \mathrm{kHz}$, and the bottom spectrum was obtained using a frequency offset of $2 \mathrm{kHz}$ and $d_{\mathrm{HH}}=10 \mathrm{kHz}$. The dotted line indicates the splitting expected from the zeroth-order average Hamiltonian theory. when the residual dipolar tensors remain symmetric and the effective angle between $\mathrm{C}-\mathrm{H}$ bonds is $0^{\circ}$ (or $180^{\circ}$ ). For instance, this situation frequently occurs in liquid crystals and lipid membranes due to fast axial motions. ${ }^{5,20-23}$ Simulated spectra with and without homonuclear proton couplings and resonance frequency offset are compared in Fig. 10 for the BB-BLEW12 spin-lock sequence. The dipolar splitting changes within $5 \%$ for practical values of the proton offset. In addition, the linewidth increases in the presence of homonuclear couplings.

\section{EXPERIMENTAL RESULTS AND DISCUSSION}

\section{A. NAVL single crystal}

The performance of the BB-BLEW12 spin-lock technique was demonstrated on a rigid solid sample of the NAVL single crystal at an arbitrary orientation relative to the external magnetic field. In this sample, ${ }^{15} \mathrm{~N}$ chemical shift spectrum exhibit, in general, four peaks corresponding to two inequivalent molecular positions in the crystal lattice. The ${ }^{15} \mathrm{~N}-{ }^{1} \mathrm{H}$ dipolar coupling varies according to the orientation of the $\mathrm{N}-\mathrm{H}$ vector for different crystal sites. In Fig. 11 the dipolar slices from 2D BB-BLEW12 spin-lock spectra are shown for the ${ }^{15} \mathrm{~N}$ sites with the largest and smallest dipolar splittings. Spectra measured at a zero proton offset frequency (as defined by the optimum decoupler frequency) and $6 \mathrm{kHz}$ offset are compared. Linewidths and intensities are practically unaffected by the offset. The value of the smaller splitting is essentially the same in the two spectra, while the larger splitting decreases by $5 \%$, in agreement with the theoretical prediction. 


\section{B. THE6}

Anisotropic fluids such as liquid crystals exhibit, typically, a wide range of residual one-bond ${ }^{1} \mathrm{H}-{ }^{13} \mathrm{C}$ interactions for carbon sites along the molecule. The application of the $\mathrm{WIM} z$ SLF experiment is demonstrated on a columnar thermotropic phase formed by discotic mesogen THE6 (Fig. 12). For the demonstration of the pulse sequence, the $\mathrm{CH}$ pair (at $\mathrm{C}_{4}$ site in the molecular core) with a relatively large dipolar coupling [Fig. 12(a)] and methylene $\mathrm{CH}_{2}$ site $(\gamma$-carbon in the side chain) with a much smaller coupling [Fig. 12(b)] were selected. Spectral shapes obtained at zero and $6 \mathrm{kHz}{ }^{1} \mathrm{H}$ resonance offsets are compared. It is obvious that for both sites the offset effect on the line shape is minor: the linewidths and intensities are virtually unchanged and splittings vary only slightly (by $3.5 \%$ and $1.5 \%$ for $\mathrm{C} 4$ and $\mathrm{C}_{\gamma}$, respectively). Analogous behavior was observed when the ${ }^{13} \mathrm{C}$ frequency was shifted. Highly resolved dipolar coupling spectra with a similar offset dependence were also obtained for the other sites in the THE6 molecule. The dependence of the splitting on the proton frequency offset shown in Fig. 12(c) is rather flat. The obtained coupling constants agree well with those previously estimated by other methods. ${ }^{18}$

\section{Comparison to other techniques}

A number of separated local field techniques applicable to the static samples have in the past been reported. ${ }^{1,6,24}$ For example, the PISEMA and proton detected local field (PDLF) experiments are popular techniques in studies of biomolecules in membranes ${ }^{3,4}$ and liquid crystals, ${ }^{2}$ respectively. PISEMA (Ref. 6) experiment is based on the spin exchange ${ }^{11}$ combined with the flip-flop Lee-Goldburg irradiation ${ }^{25,26}$ for homonuclear proton decoupling. Due to a short rf cycle time, it is well suited for the measurement of large dipolar couplings. The PDLF technique involves the evolution of the transverse magnetization of protons coupled to rare nuclei. Because of a long rf cycle time of the homonuclear decoupling sequence used in PDLF and the requirement for the refocusing of chemical shift, the experiment results in a limited width of the spectral window in the indirect dimension and therefore is mainly used for studies on mobile systems where dipolar couplings are significantly attenuated by anisotropic molecular motions.

Dipolar spectra obtained in NAVL single crystal by WIM and PISEMA techniques are compared in Fig. 13. The resolution of large dipolar couplings is comparable for two techniques. For smaller couplings, WIM-based HIMSELF experiment produces better resolved and less distorted peaks. It is known that it is difficult to accurately measure small couplings by PISEMA due to strong interference effect from the proton chemical shift interaction. ${ }^{5,14}$ In contrast, the WIM technique provides rather uniform resolution irrespective of the dipolar coupling value.

Figure 14 compares the dipolar spectra obtained for different carbon sites of a columnar liquid crystalline phase of THE6. Dipolar coupling is considerably reduced in this sample due to anisotropic rotation and conformation mobility of molecules. Therefore, it was also possible to apply SLF
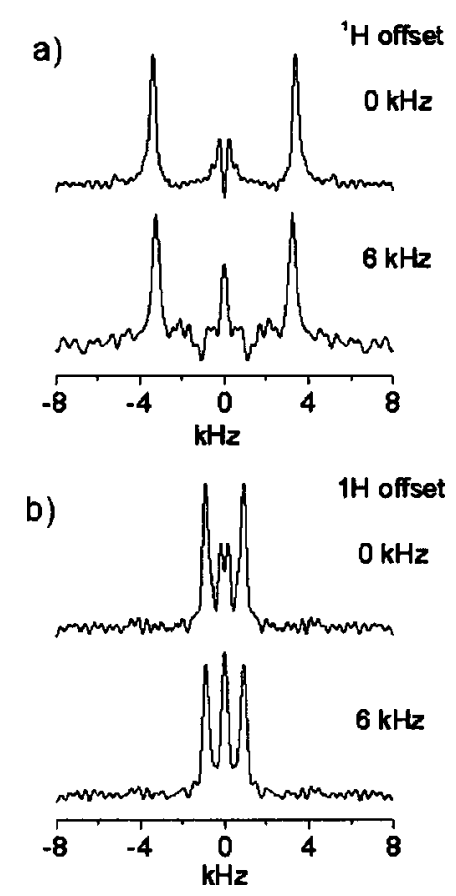

FIG. 11. BB-BLEW12 spin-lock ${ }^{15} \mathrm{~N}-{ }^{1} \mathrm{H}$ dipolar spectra from NAVL single crystal at an arbitrary orientation relative to the external magnetic field. Spectra for the inequivalent sites with the largest (a) and smallest (b) dipolar splittings are shown. Spectra measured at a zero proton offset frequency (as defined by the optimal decoupler frequency) and $6 \mathrm{kHz}$ offset are compared.

and PDLF sequences employing a long and highly efficient homonuclear decoupling sequence like BLEW48.

The splittings obtained from different techniques are consistent within experimental errors. As expected, the spectra obtained using conventional SLF techniques exhibit the least dipolar resolution. PDLF technique provides a superior resolution for most of the sites. It is also possible to detect the $\mathrm{C}-\mathrm{H}$ couplings between remote spins in PDLF experiments. To collect PISEMA data, due to strong effects of the proton chemical shift on the dipolar coupling evolution in this technique, two experiments were carried out with the proton frequency offset optimized either for the aliphatic or aromatic molecular sites. This allowed obtaining a well resolved spectra for most of the sites with a relatively high splitting, while for the sites with a small splitting, a significant distortion of spectral shapes was observed. WIM spectra demonstrate a uniform resolution irrespective of the strength of the dipolar coupling and chemical shift.

In general, PISEMA is the method of choice for the measurement of large dipolar couplings. For the measurement of smaller dipolar couplings, in spite of the high dipolar scaling factor of this sequence, significant errors are present due to the strong effect of the offset. A modified version of the basic PISEMA experiment, BB-PISEMA, was recently reported, ${ }^{27,28}$ where the offset effect for the smaller coupling is significantly reduced. On the other hand, for the measurement of a smaller dipolar coupling, where a wide spectral window in the dipolar dimension is not required, the PDLF technique involving the long rf cycle homonuclear decoupling sequence can be used. The long increment time in the dipolar dimension for this approach is due to both a long rf cycle and the need for chemical shift refocusing delay. WIM 
a)

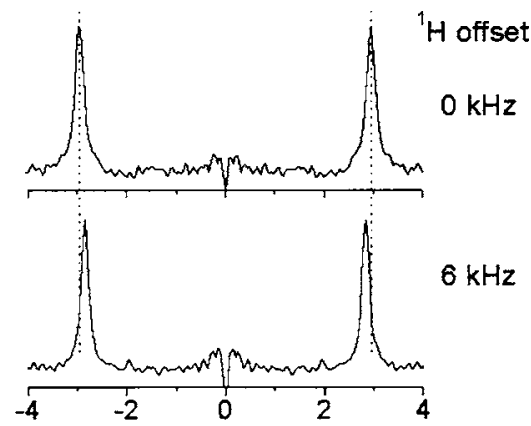

b)

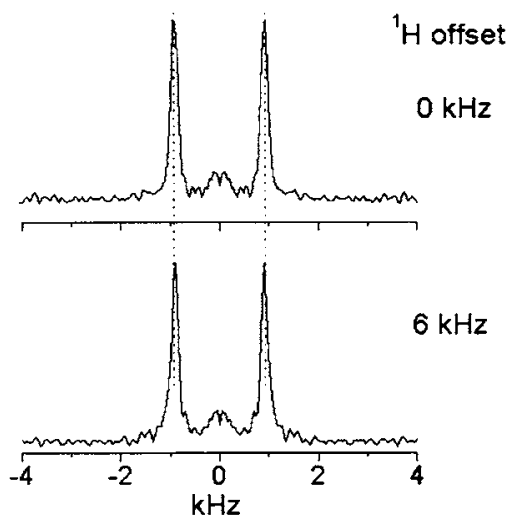

c)

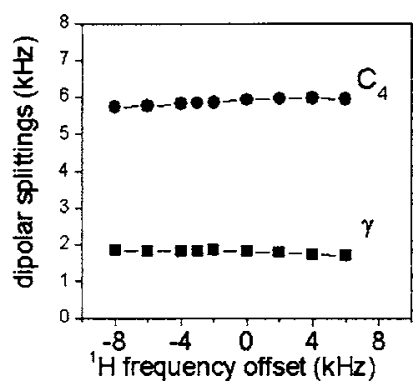

FIG. 12. WIMz-based HIMSELF spectra in a columnar thermotropic phase of mesogen THE6 at $85{ }^{\circ} \mathrm{C}$. (a) $\mathrm{CH}$ pair corresponding to the $\mathrm{C}_{4}$ site of the molecular core. (b) Methylene $\gamma-\mathrm{CH}_{2}$ site. Spectral shapes obtained at zero and $6 \mathrm{kHz}{ }^{1} \mathrm{H}$ resonance offsets are compared. (c) The dependence of the dipolar splitting on the proton frequency offset.

and BLEW techniques offer a reasonable compromise for the whole range of the dipolar coupling values irrespective of the chemical shift frequency. High dipolar resolution comparable to that of PISEMA is also achieved. Moreover, while the use of a high rf power for the flip-flop Lee-Goldburg (FFLG) spin-lock sequence suppresses the offset effects and dramatically improves resolution of PISEMA, it increases the required rf power in the $S$ spin channel. For example, several hundred Watts of rf power are required for ${ }^{13} \mathrm{C}$ nuclei, while about a kilowatt rf power is required for ${ }^{15} \mathrm{~N}$ nuclei. This high power is not suitable for heat sensitive samples such as wet biological solids and liquid crystalline materials. Therefore, low power PISEMA-type experiments, called PITANSEMA (polarization inversion time averaged nutation spin exchange at the magic angle), were developed using the time averaged nutation concept. ${ }^{29-31}$ These experiments were successfully used to study thiophene-based functional thermotropic liquid crystalline samples. ${ }^{32,33}$ However, the use of low rf power in PITANSEMA experiments is not suitable to suppress the offset effects. On the other hand, the on-

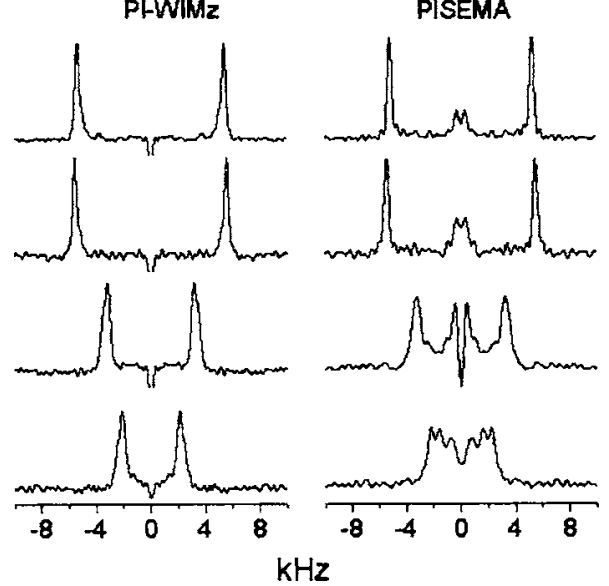

FIG. 13. Dipolar spectra obtained from NAVL single crystal using WIM and PISEMA techniques. The slices for four inequivalent crystal sites are shown. Frequency scales are corrected for the scaling factors of 0.67 and 0.82 for WIM and PISEMA, respectively.

resonance broadband HIMSELF sequences based on BLEW and WIM that overcome the offset effects will also be useful to study the heat sensitive samples as they utilize less rf power than the PISEMA sequence.

Another advantage of BLEW spin-lock and WIM approaches, as compared to other methods discussed above, is that the homonuclear spin decoupling is obtained in both frequency channels. Therefore these techniques can be applied to uniformly ${ }^{13} \mathrm{C}$ or ${ }^{15} \mathrm{~N}$ labeled samples, where significant homonuclear dipolar interactions between $S$ nuclei might be present.

\section{CONCLUSIONS}

In this paper we have theoretically, numerically, and experimentally analyzed the performance of HIMSELF techniques based on windowless homonuclear decoupling sequences. WIM and BLEW spin-lock types of the separated local field experiments are useful in the systems where a wide range of the dipolar coupling constants and the significant chemical shift dispersion are present. In such a case these techniques offer uniform dipolar resolution for both large and small dipolar couplings. In contrast to PISEMA, the chemical shift and frequency offset terms are significantly suppressed. In addition, due to its ability to suppress the homonuclear spin interactions for both spin species, these methods can be applied to uniformly labeled compounds. Because of the isotropic mixing property of the dipolar coupling Hamiltonian effective during these sequences, new methods provide a great flexibility in the design of the multidimensional experiments. For example, the transfer of the longitudinal spin polarization can be performed. For highly mobile liquid crystals exhibiting a relatively small dipolar coupling, the greatest dipolar resolution is achieved using the PDLF approach. On the other hand, for the immobilized oriented molecules, as, for instance, the peptides and proteins in biomembranes, where the full range of the dipolar coupling constants is present due to distribution of the bond orientation in different residues, the new technique could be advantageous. Therefore, we believe that these new pulse se- 

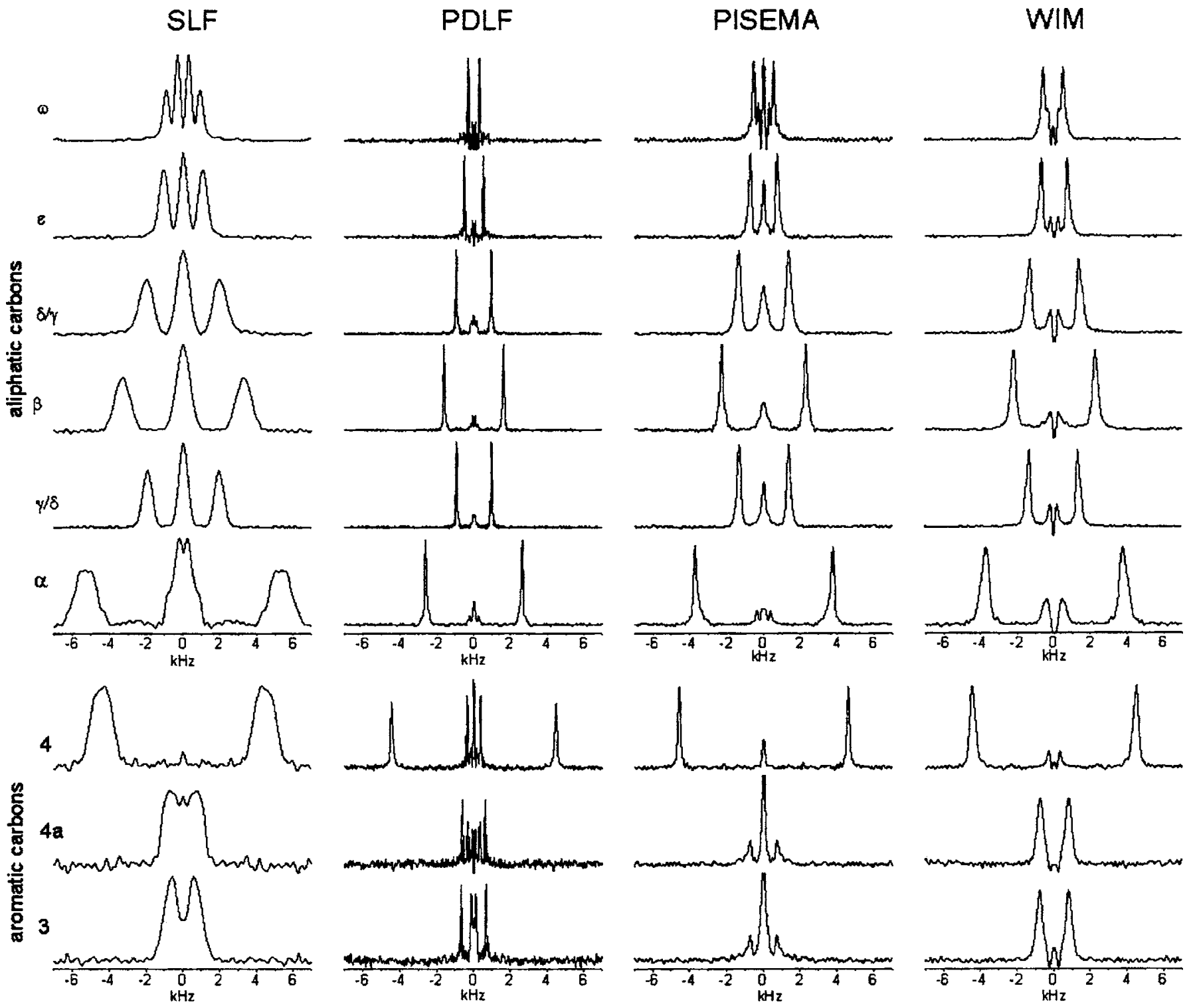

FIG. 14. Comparison of the dipolar spectra obtained in a columnar mesophase of THE6 at $85{ }^{\circ} \mathrm{C}$ by SLF, PDLF, PISEMA, and WIM techniques. The slices for four chemically inequivalent sites in the molecule are shown. Frequency scales are corrected for scaling factors of $0.42,0.42,0.82$, and 0.67 for SLF, PDLF, PISEMA and WIM sequences, respectively.

quences will also be useful in improving the existing multidimensional methods ${ }^{4,34-37}$ to study biological solids.

\section{ACKNOWLEDGMENTS}

The authors thank Dr. Herbert Zimmermann for providing the liquid crystal. This work was supported by the National Institutes of Health (AI054515) and the Russian Foundation for Basic Research (Grant No. 04-03-32639).

${ }^{1}$ R. K. Hester, J. L. Ackerman, B. L. Neff, and J. S. Waugh, Phys. Rev. Lett. 36, 1081 (1976).

${ }^{2}$ S. Caldarelli, in Encyclopedia of Nuclear Magnetic Resonance, edited by D. M. Grant and R. K. Harris (Wiley, Chichester, 2002), p. 291

${ }^{3}$ S. J. Opella, in Encyclopedia of Nuclear Magnetic Resonance, edited by D. M. Grant and R. K. Harris (Wiley, Chichester, 2002), p. 427

${ }^{4}$ A. Ramamoorthy, Y. Wei, and D.-K. Lee, Annu. Rep. NMR Spectrosc. 52, 1 (2004).

${ }^{5}$ S. V. Dvinskikh, H. Zimmermann, A. Maliniak, and D. Sandström, J. Magn. Reson. 163, 46 (2003).

${ }^{6}$ C. H. Wu, A. Ramamoorthy, and S. J. Opella, J. Magn. Reson., Ser. A
109, 270 (1994); A. Ramamoorthy, C. H. Wu, and S. J. Opella, J. Magn. Reson. 140, 131 (1999); A. Ramamoorthy and S. J. Opella, Solid State NMR Spectrosc 4, 387 (1995).

${ }^{7}$ S. V. Dvinskikh, K. Yamamoto, and A. Ramamoorthy, Chem. Phys. Lett. 419, 168 (2006).

${ }^{8}$ K. Yamamoto, S. V. Dvinskikh, and A. Ramamoorthy, Chem. Phys. Lett. 419, 533 (2006).

${ }^{9}$ D. P. Burum, M. Linder, and R. R. Ernst, J. Magn. Reson. (1969-1992) 44, 173 (1981).

${ }^{10}$ P. Caravatti, L. Braunschweiler, and R. R. Ernst, Chem. Phys. Lett. 100, 305 (1983).

${ }^{11}$ S. R. Hartmann and E. L. Hahn, Phys. Rev. 128, 2042 (1962).

${ }^{12}$ R. R. Ernst, G. Bodenhausen, and A. Wokaun, Principles of Nuclear Magnetic Resonance in One and Two Dimensions (Clarendon, Oxford, 1987).

${ }^{13}$ J. Cavanagh, W. J. Fairbrother, A. G. Palmer, and N. J. Skelton, Protein NMR Spectroscopy (Academic, San Diego, 1996).

${ }^{14}$ Z. Gan, J. Magn. Reson. 143, 136 (2000).

${ }^{15}$ A. J. Shaka, in Encyclopedia of Nuclear Magnetic Resonance, edited by D. M. Grant and R. K. Harris (Wiley, New York, 1996), p. 1558.

${ }^{16}$ R. Fu, C. Tian, H. Kim, S. A. Smith, and T. A. Cross, J. Magn. Reson. 159, 167 (2002).

${ }^{17}$ C. Counsell, M. H. Levitt, and R. R. Ernst, J. Magn. Reson. (1969-1992) 
63, 133 (1985).

${ }^{18}$ D. Goldfarb, Z. Luz, and H. Zimmermann, J. Chem. Phys. 78, 7065 (1983).

${ }^{19}$ M. Bak, J. T. Rasmussen, and N. C. Nielsen, J. Magn. Reson. 147, 296 (2000).

${ }^{20}$ S. V. Dvinskikh, Z. Luz, H. Zimmermann, A. Maliniak, and D. Sandström, J. Phys. Chem. B 107, 1969 (2003).

${ }^{21}$ S. V. Dvinskikh, D. Sandström, H. Zimmermann, and A. Maliniak, Chem. Phys. Lett. 382, 410 (2003).

${ }^{22}$ S. V. Dvinskikh, V. Castro, and D. Sandström, Phys. Chem. Chem. Phys. 7, 607 (2005).

${ }^{23}$ S. V. Dvinskikh, V. Castro, and D. Sandström, Phys. Chem. Chem. Phys. 7, 3255 (2005).

${ }^{24}$ P. Caravatti, G. Bodenhausen, and R. R. Ernst, Chem. Phys. Lett. 89, 363 (1982).

${ }^{25}$ M. Mehring and J. S. Waugh, Phys. Rev. B 5, 3459 (1972).

${ }^{26}$ A. Bielecki, A. C. Kolbert, and M. H. Levitt, Chem. Phys. Lett. 155, 341 (1989).

${ }^{27}$ S. V. Dvinskikh and D. Sandström, J. Magn. Reson. 175, 163 (2005).
${ }^{28}$ K. Yamamoto, D. K. Lee, and A. Ramamoorthy, Chem. Phys. Lett. 407, 289 (2005).

${ }^{29}$ D. K. Lee, T. Narasimhaswamy, and A. Ramamoorthy, Chem. Phys. Lett. 399, 359 (2004).

${ }^{30}$ K. Nishimura and A. Naito, Chem. Phys. Lett. 402, 245 (2005).

${ }^{31}$ K. Yamamoto, V. L. Ermakov, D. K. Lee, and A. Ramamoorthy, Chem. Phys. Lett. 408, 118 (2005).

${ }^{32}$ T. Narasimhaswamy, D. K. Lee, K. Yamamoto, N. Somanathan, and A. Ramamoorthy, J. Am. Chem. Soc. 127, 6958 (2005).

${ }^{33}$ T. Narasimhaswamy, M. Monette, D. K. Lee, and A. Ramamoorthy, J. Phys. Chem. B 109, 19696 (2005).

${ }^{34}$ A. Ramamoorthy, C. H. Wu, and S. J. Opella, J. Magn. Reson., Ser. B 107, 88 (1995)

${ }^{35}$ A. Ramamoorthy, L. M. Gierasch, and S. J. Opella, J. Magn. Reson., Ser. B 109, 112 (1995)

${ }^{36}$ A. Ramamoorthy, L. M. Gierasch, and S. J. Opella, J. Magn. Reson., Ser. B 111, 81 (1996).

${ }^{37}$ A. Ramamoorthy, L. M. Gierasch, and S. J. Opella, J. Magn. Reson., Ser. B 110, 102 (1996). 